\title{
RSOA-Based Wavelength-Reuse Gigabit WDM-PON
}

\author{
Byoung Whi Kim* \\ WDM-PON Technology Team, Electronics and Telecommunications Research Institute, 161 \\ Gajeong-dong, Yuseong-gu, Daejeon, Korea
}

(Received November 13, 2008 : revised December 9, 2008 : accepted December 9, 2008)

\begin{abstract}
This article presents the RSOA-based re-modulation schemes for practical application to the WDM-PON link. Emphasis is put on the three methods for reducing the residual downstream signal in the upstream transmission; the simplest one is to use the RSOA gain-saturation property, the second uses selective filtering of the adiabatic-chirped DFB-LD output spectrum, and the last uses a dynamic RSOA gain control by varying its driving current according to the incident binary signal. The reflection sensitivity in the proposed re-modulation link is also qualitatively presented in the cases of using two different types of seed light: coherent light from DFB-LD array and spectrum-sliced incoherent ASE light. We show experimental results of an error-free bidirectional gigabit transmission over $20 \mathrm{~km}$ at $-30 \mathrm{~dB}$ reflection with the spectrum-sliced ASE seed light.
\end{abstract}

Keywords: WDM-PON, RSOA, Re-modulation

OCIS codes : (060.0060) Fiber optics and optical communications; (060.4230) Multiplexing; (060.4250) Networks; (060.4510) Optical communications

\section{INTRODUCTION}

The recent changes in paradigm of services through the network have stimulated the network providers to find out viable technologies that should be able to fulfill the requirements of future-proof bandwidth provisioning, and convergence of broadcasting and telecommunication services [1-2]. Among the various solutions to the FTTH realization, the wavelengthdivision-multiplexed passive optical network (WDMPON) has been considered as an ultimate next-generation solution because of its large transmission capacity, network security, and data transparency.

However, for the practical deployment of WDM-PON systems, the optical network terminal (ONT) placed in the subscriber premises should be wavelength-independent or wavelength-free, in addition to being cost-competitive to existing technologies. To this end, many sophisticated solutions have been reported in the literature. In the spectrum slicing scheme [3], the optical source is a broadband light source (BLS) and its output spectrum is spectrally sliced by the wavelength filter placed at OLT or remote node. However this scheme has limitations

*Corresponding author: kbw@etri.re.kr in extending the transmission distance up to $20 \mathrm{~km}$ and increasing the data rate over $1 \mathrm{~Gb} / \mathrm{s}$ due to both the spectrum slicing-induced noise increase and a huge power loss (typically higher than $15 \mathrm{~dB}$ ) during the spectrum slicing.

Another is the injection-locking scheme [4-5]. Later on, this scheme is renamed as wavelength-locking because the laser diode output light is locked in wavelength to the incident seed light. Here, it uses a speciallydesigned Fabry-Perot laser diode (FP LD) for providing full colorless operation within its operational wavelength region. The WDM-PON of this scheme with a couple of BLSs being used as spectrum-sliced external injector has been successfully deployed to Fiber To the Curb (FTTC) networks by Korea Telecom (KT), providing $100 \mathrm{Mb} / \mathrm{s}$ per wavelength and 32 wavelength-multiplexed on a fiber [6]. However, it would not be easy for the scheme to provide higher data rates beyond $1 \mathrm{~Gb} / \mathrm{s}$ over longer than $20 \mathrm{~km}$ distance due to the slicing-induced noise and the mode-partition noise. Alternatively, to suppress mode-partition noise, it has been proposed to use a reflective semiconductor optical amplifier (RSOA) instead of FP-LDs [7]. Also, for the enhancement of noise characteristics, a BLS can be replaced by a single-mode laser array [8]. 


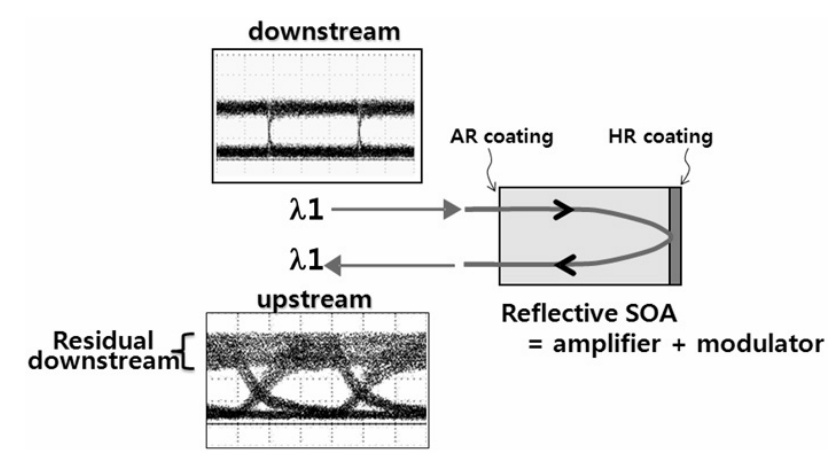

FIG. 1. Re-modulation (RM) scheme by employing a RSOA as a colorless transmitter.

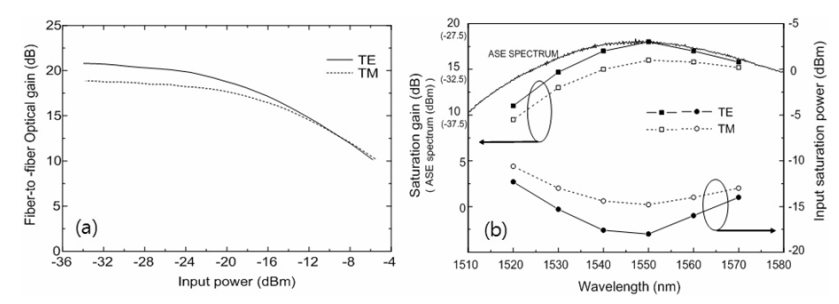

FIG. 2. Optical gain values of TO-packaged RSOA module with respect to (a) the input optical power and (b) the wavelength of the incident light (Ref. [15]).

Although these ideas have attracted much attention over the years and especially the injection- or wavelengthlocking scheme has been already commercialized, further efforts have been placed to increase transmission speed per wavelength and, at the same time, to simplify the link structure by eliminating light sources such as BLS which were used in addition to the light sources used for carrying the data. As a part of the efforts, the remodulation (RM) schemes in which the downstream optical signal is reused for upstream transmission have been developed, based on the usage of an RSOA as a colorless source [9-15]. As shown in Fig. 1, the RSOA in the RM schemes play roles of both amplifying the downstream signal and modulating it with upstream data.

The biggest problem in the RM schemes is the imperfect erasing of the modulated input light, eventually resulting in the residual downstream signal in the upstream "1"-level, as shown in the upstream eye pattern in Fig. 1. The amount of this residue from the imperfection is proportional to the extinction ratio (ER) of the downstream optical signal. As this issue should be of critical importance in deciding whether the schemes can be practically applicable to the real field, we spend a significant portion of the article in addressing how we can overcome this problem.

In this paper, firstly we review the gain-saturation characteristics and its related properties such as suppression of the downstream ER, noise reduction at low frequency region, and nonlinear spectral distortion. And then, we explain our novel optical/electrical compensa- tion techniques such as frequency-detuning and feedforward current injection. Improved transmission results are presented especially in terms of the increase of an allowable downstream ER. The reflection sensitivity in the proposed re-modulated links is qualitatively presented in the cases of using two different types of seed lights: coherent light from DFB-LD array and spectrumsliced ASE light. Finally, conclusive remarks are given.

\section{GAIN-SATURATION of THE RSOA}

\section{A. Gain-Saturation}

In the gain-saturation regime, the optical gain in an RSOA decreases as the injection optical power increases. Here the gain means the ratio of output optical power to the input one. The input saturation optical power, $\mathrm{P}_{\text {in/sat }}$, is usually defined as the input optical power at which the RSOA gain drops by $3 \mathrm{~dB}$ from its small signal gain [15]. The saturation gain, $\mathrm{G}_{\mathrm{s}}$, is the gain at the input optical power of $\mathrm{P}_{\text {in/sat }}$.

As an illustration on the current status of an RSOA gain performance, Fig. 2(a) shows measured values of the polarization-dependent fiber-to-fiber optical gain of the TO-packaged RSOA at the operating current of 60 $\mathrm{mA}$. Also, the gain saturation characteristic depends on the wavelength of the injected light, which is closely related to the amplified spontaneous emission (ASE) spectrum of the RSOA. The wavelength-dependent characteristics of Gs and $\mathrm{P}_{\text {in/sat }}$ are shown in Fig. 2(b). The ASE spectrum is also plotted as a reference. The 3 -dB bandwidth (BW) of the ASE spectrum is around $53 \mathrm{~nm}$. It is noted that the wavelength dependence of Gs follows the ASE spectral shape, which has 3-dB gain BW fully covering 32 WDM channels with $100-\mathrm{GHz}$ channel spacing. On the other hand, the wavelengthdependent behavior of $\mathrm{P}_{\text {in/sat }}$ is opposite to that of Gs. It implies that the RSOA gain near the ASE center wavelength begins to saturate at the lower injection power. Over the wavelengths from $1530 \mathrm{~nm}$ to $1560 \mathrm{~nm}$, the smallest value of $\mathrm{G}_{\mathrm{s}}$ is around $13 \mathrm{~dB}$, and the largest value of $\mathrm{P}_{\text {in/sat }}$ is $-13 \mathrm{dBm}$ at $1530 \mathrm{~nm}$.

\section{B. Reduction of Extinction Ratio in Gain-Saturation Regime}

The easiest way of suppressing the downstream data pattern included in the upstream signal may be using the gain-saturation characteristic of the RSOA. In a gain-saturation regime, the optical gain at the lower injection power is higher than that at the higher injection power. This gain difference between "0"-level and "1"-level results in a suppression of the downstream ER. It becomes pronounced as the injection optical power to an RSOA increases (deep saturation regime), providing a better ER compensation. The measured 


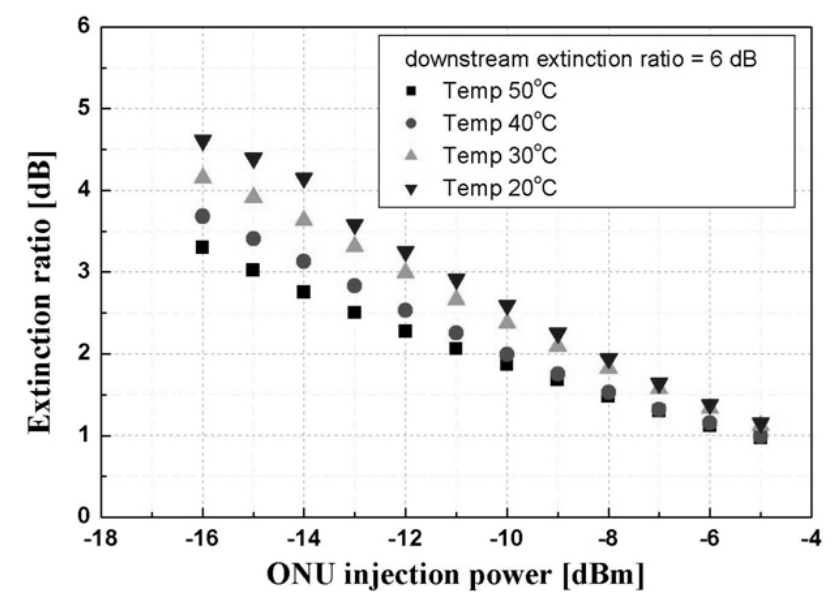

FIG. 3. Measured extinction ratio (ER) of the output light from an RSOA when the incident ER is $6 \mathrm{~dB}$. The DC driving current to an RSOA is $60 \mathrm{~mA}$ (not modulated).

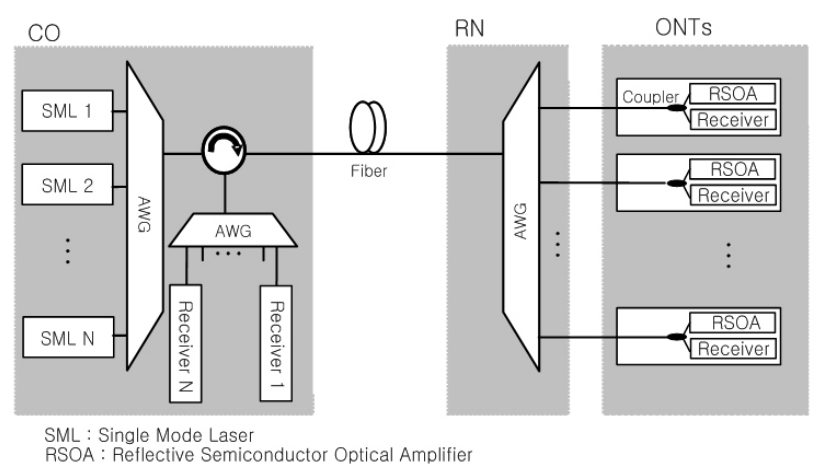

FIG. 4. Re-modulation-based WDM-PON structure employing RSOAs (Ref. [15])

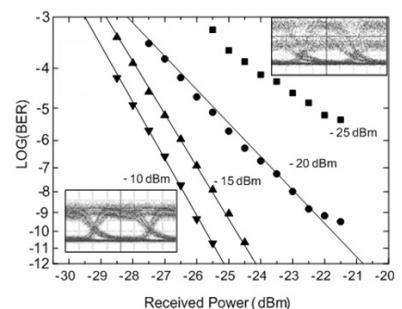

(a)

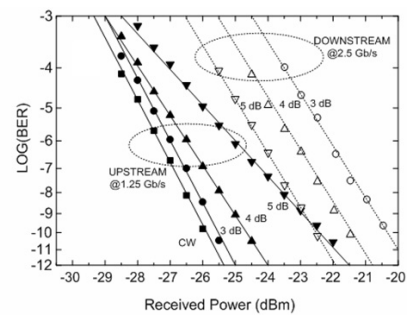

(b)
FIG. 5. (a) Measured back-to-back BER plots with respect to the optical power injected into an RSOA. The downstream ER is $4 \mathrm{~dB}$. (b) Measured BER performances over $20 \mathrm{~km}$ distance with several values of downstream ERs. The injected power to the RSOA is $-15 \mathrm{dBm}$ (ref. $[15])$.

ER values of the RSOA output light at the input ER value of $6 \mathrm{~dB}$ are plotted in Fig. 3 by varying the input optical power level. As expected, the output ER value decreases as the input power increases or as the RSOA goes into a deeper saturation regime.

Also the magnitude of the ER reduction depends on the RSOA operating temperature. As in the semicon- ductor laser diode, the RSOA optical power and its gain decrease with the temperature. At a low injection power of $-18 \mathrm{dBm}$, the resulting ER difference between $50^{\circ} \mathrm{C}$ and $20^{\circ} \mathrm{C}$ is $1.5 \mathrm{~dB}$. However, this ER difference becomes $0.2 \mathrm{~dB}$ at a high injection power of $-5 \mathrm{dBm}$. It means that an un-cooled or temperature-independent operation of an RSOA may be allowed in the case of a high power injection. However, considering a practical link loss budget and an available light source, the allowed injection power level in the RM scheme would be around $-15 \mathrm{dBm}$, which is much lower than the required power for an un-cooled RSOA operation. Hence, for practical implementation, the RSOA transmitter should be able to keep the RSOA temperature constant in an uncontrolled environment.

\section{Transmission Results}

To investigate the transmission performance in the RM scheme using RSOA's gain-saturation, we measured the bit-error ratios (BERs) for both upstream and downstream with various conditions. The link structure of interest is schematically illustrated in Fig. 4. For downstream transmission, a 1550-nm DFB-LD was directly modulated with 2.5-Gb/s non-return-to-zero data. With this downstream signal as a seed light to an RSOA, the upstream signal is generated by direct modulation of an RSOA with $1.25-\mathrm{Gb} / \mathrm{s}$ NRZ data $\left(2^{7}-1\right.$ PRBS). We used an optical receiver employing a PIN photodiode for measuring BER data both upstream and downstream.

Figure 5(a) shows the measured BER for back-to-back upstream transmission with varying the RSOA injected power levels from $-25 \mathrm{dBm}$ to $-10 \mathrm{dBm}$, i.e., from the linear regime to the gain saturation regime. The optical eye patterns corresponding to the RSOA injection powers of $-10 \mathrm{dBm}$ and $-25 \mathrm{dBm}$ are also included in the insets of Fig. 5(a). The higher the injection power into the RSOA is, the more suppressed the downstream signal residue in the upstream signal becomes, eventually enhancing the BER performance of upstream. We also investigated the effect of the downstream ER on the BER performances for both upstream and downstream transmission over $20-\mathrm{km}$ single-mode fiber, as shown in Fig. 5(b). We were able to achieve an error-free upstream transmission with the downstream ER of up to $5 \mathrm{~dB}$; the difference in sensitivity between back-to-back and $20-\mathrm{km}$ single-mode fiber is negligibly small. However, as expected, we clearly observed deterioration of the upstream performance as the downstream ER increases [15].

The downstream ER-induced power penalty can be lowered by increasing the optical power injected into the RSOA. However, the maximum injection power is limited by the overall link power budget and output power of the OLT transmitter. Since the downstream ER lower than $5 \mathrm{~dB}$ leads to additional power penalty of more than $3 \mathrm{~dB}$ in the downstream transmission, the 


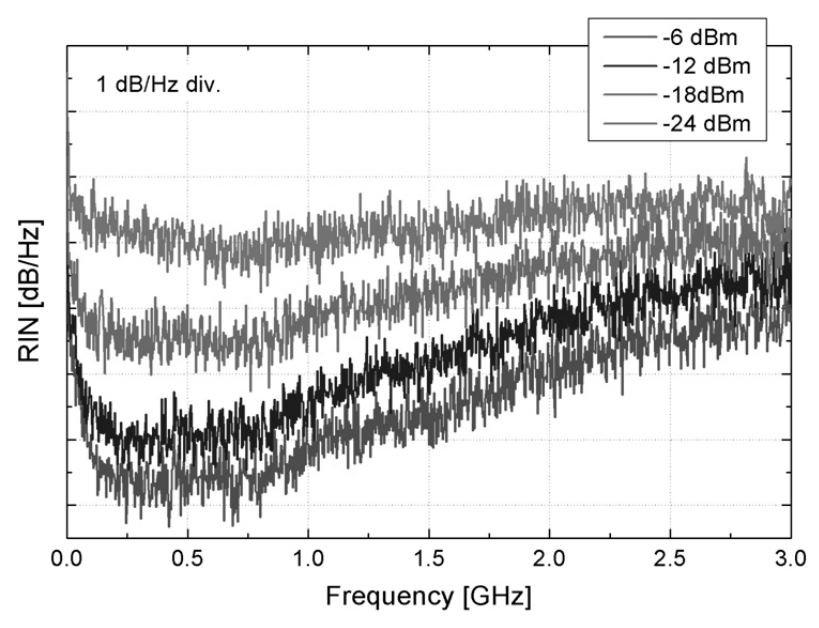

FIG. 6. Measured RIN of the RSOA output light when a spectrum-sliced ASE light is injected to an RSOA.

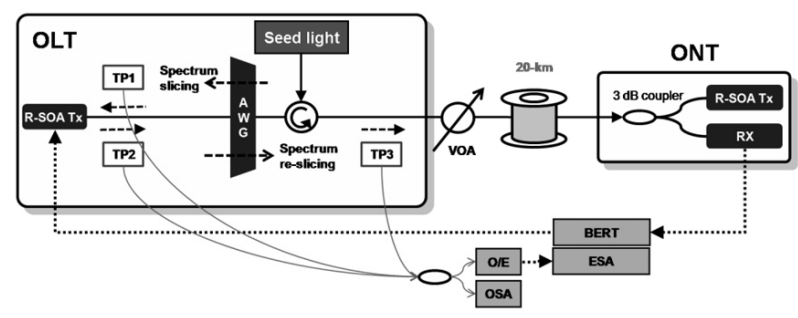

FIG. 7. Experiment setup for measuring spectral distortion when a spectrum-sliced ASE light is injected to an RSOA.
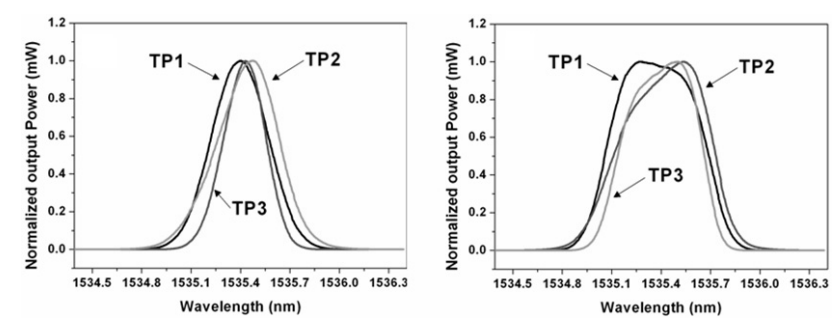

FIG. 8. Normalized optical spectra at the TP1, TP2 and TP3 with an AWG filter. (a) Gaussian-shaped pass-band AWG and (b) flat-top pass-band AWG.

downstream ER should be carefully chosen, taking into account both power penalties of the upstream and downstream transmission.

\section{Noise Reduction of Spectrum-Sliced ASE Light}

Besides a low-noise coherent light such as DFB-LD output light, it is possible to use a spectrum-sliced ASE (SSA) light as a seed light to an RSOA in the RM schemes. However, the relative-intensity-noise (RIN) level of the SSA light is around $-100 \mathrm{~dB} / \mathrm{Hz}$, much worse than that in a DFB-LD. With this noise level, it is not possible to achieve an error-free gigabit transmission over $20 \mathrm{~km}$ of single-mode fiber.

One way of lowering the RIN of the SSA light is to use the RSOA gain-saturation property where it acts as a high-frequency pass filter [16]. For a data rate of 1.25 Gbps with the nominal receiver $3-\mathrm{dB}$ BW of around $900 \mathrm{MHz}$, the noise frequency region which is important in determining the signal-to-noise ratio is up to $1 \mathrm{GHz}$. Fortunately, when the SSA light is injected to a gainsaturated RSOA, the intensity noise in the lower frequency region (especially less than $1 \mathrm{GHz}$ ) is reduced to such a level of achieving an error-free gigabit transmission [17].

As in the case of the ER reduction, the magnitude of RIN reduction also depends on how much RSOA gain becomes saturated. As an illustration of dependence of the RIN reduction on the injection SSA power, we plot the measured intensity noise in Fig. 6 by varying the injection power of the SSA light. As expected, the noise reduction at less than $1 \mathrm{GHz}$ is clearly seen when the injection power is higher than $-12 \mathrm{dBm}$. In comparison to the RIN value of the SSA light itself, the noise power is reduced by around $10 \mathrm{~dB}$.

It should be noted that the RSOA operation in a gainsaturation regime is not an efficient method in reducing the noise at a frequency region higher than $2 \mathrm{GHz}$. We expect that it would impose a fundamental limit on an allowable data rate if there were no other viable methods of noise reduction of the SSA light.

\section{E. Noise Reduction and Spectral Distortion}

The aforementioned noise reduction of the SSA light in a gain-saturated RSOA accompanies nonlinear spectral distortion where the RSOA output spectrum shape is pretty much different or spectrally distorted from the originally injected one [18].

For investigating this spectral distortion in the experimental setup of Fig. 7, we measure the optical spectrum at three test-points (TPs): TP1 for the injected SSA light, TP2 for an RSOA output light and TP3 for an RSOA output light sliced again by an AWG filter. The RSOA bias current was $80 \mathrm{~mA}$ and the operating temperature was set to be $40^{\circ} \mathrm{C}$. The signal wavelength is $1535.4 \mathrm{~nm}$. Here, we used two different types of AWGs: Gaussian pass-band with 1-dB BW of $0.3 \mathrm{~nm}$ and flat-top pass-band of $1-\mathrm{dB}$ BW of $0.5 \mathrm{~nm}$. The measured optical spectra at each of the three TPs are shown in Fig. 8. Here, for clear understanding of spectral distortion, we normalize their output spectra by making its peak value to be unity in a linear scale. In both cases of using different AWGs, the output spectra from an RSOA (at TP2) are broadened and the peak wavelengths are redshifted, which is quite different from an original injected spectrum (at TP1). Consequently, after re-sliced by an AWG, the final spectrum (TP3) becomes pretty much distorted from the original one (at TP1).

The spectral distortion in a gain-saturation regime could be a serious problem in passing through several optical filters along the optical path from OLT to ONT. 


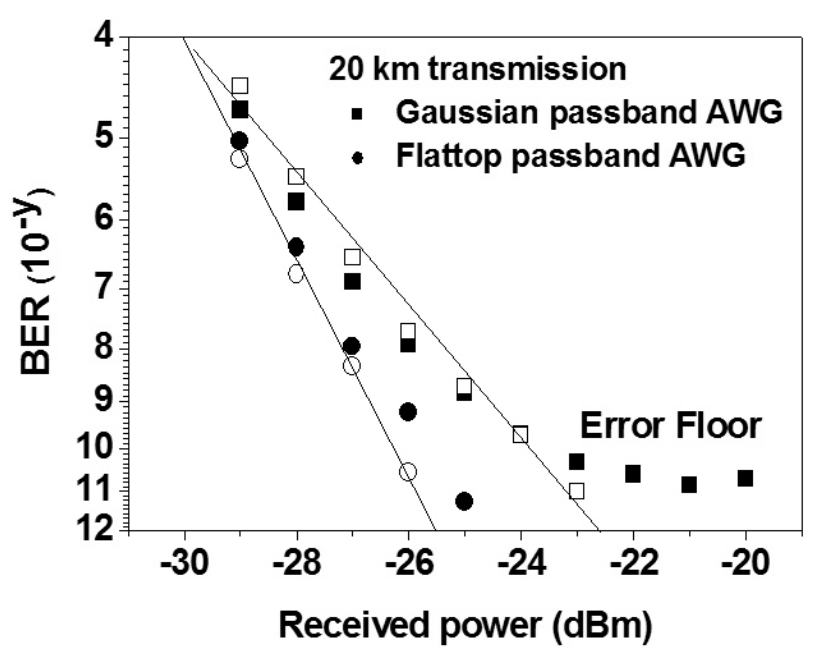

FIG. 9. Downstream BER plots with two types of AWGs. Back-to-back in an open symbol and $20 \mathrm{~km}$ transmission in a closed symbol.

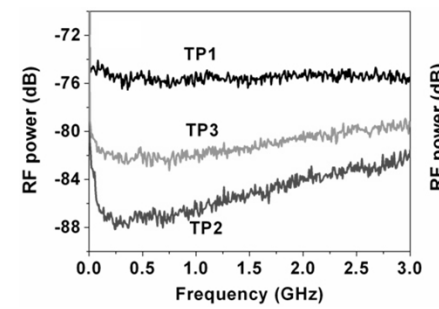

(a)

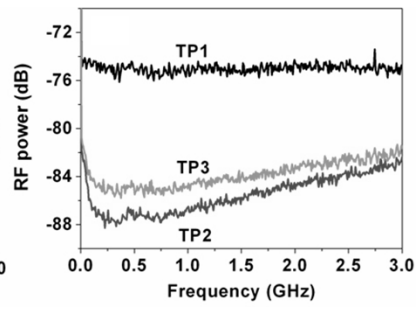

(b)
FIG. 10. Measured relative intensity noise at the TP1, TP2 and TP3 with an AWG filter. (a) Gaussian-shaped pass-band AWG and (b) flat-top pass-band AWG.

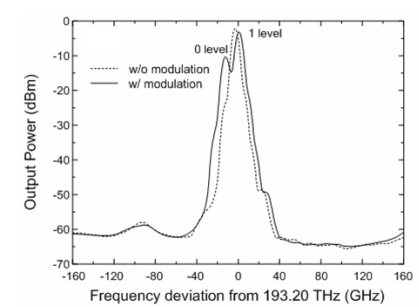

(a)

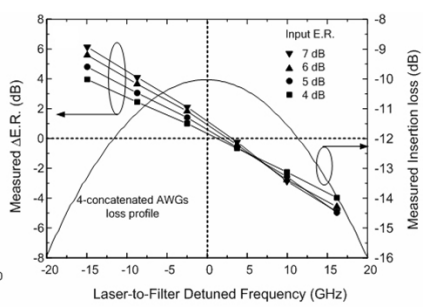

(b)
FIG. 11. (a)The optical spectrum of the adiabatic-chirped DFB-LD directly modulated with 1.25 -Gbs NRZ signal. (b)The measured values of extinction ratio variation with respect to the amount of detuned frequency between directly modulated DFB-LD and the four-concatenated filters' pass-band (Ref. [21]).

As an illustration, the measured BER plots in downstream transmission are shown in Fig. 9. The downstream ER was set to $6 \mathrm{~dB}$ in the RM schemes. The measured downstream penalty when using flat-top AWG filters was around $0.5 \mathrm{~dB}$. However, we observed an error floor with a Gaussian AWG after 20km transmission. The measured intensity noise at each test-point is plotted in Fig. 10. When using a Gaussian AWG, the intensity noise is much higher by around $3 \mathrm{~dB}$ than that with a flat-top AWG. Even though the RSOA is a good noise suppressor, the downstream signal will suffer from the spectrum re-slicing effect, as shown in Fig.10 (a) TP3.

Spectrum-broadening in the RSOA amplified SSA light is related with the facts that the RSOA linewidth broadening factor becomes high in a gain-saturation regime and that the intensity fluctuation results in carrier density fluctuation which eventually yields phase modulation [19-20].

\section{IMPROVED RE-MODULATION SCHEMES}

In a sole usage of RSOA gain-saturation for reducing the residual downstream ER in the upstream, the remote RSOA injection power should be in a deep saturation regime with the downstream ER to be less than $4 \mathrm{~dB}$. These two constraints impose a crucial limitation on practically implementing bi-directional gigabit WDMPON link. In this section, we present two elaborate methods of reducing residual downstream ER.

\section{A. Frequency Detuning}

In the RM link structure as shown in Fig. 4, the downstream signal is obtained by directly modulating the injection current to the DFB-LD at CO. Then, its output spectrum becomes adiabatic-chirp dominant and shows two local peaks which are associated respectively with the "0"-level and the "1"-level, as shown in Fig. 11(a). Its spectral separation depends on the magnitude of the modulation current and an adiabatic chirp parameter of a DFB-LD, whereas the power difference between these two peaks matches the downstream ER [21]. This downstream passes through wavelength filters four times until retuning to the upstream receiver at $\mathrm{CO}$ over its loop-back path. Hence, passing through wavelength filters repeatedly leads to the ER change because of a selective spectral filtering [22]. Figure 11(b) represents the change of measured ER values after passing through four-concatenated AWG filters. Here, the laserto-filter detuned frequency is defined as the amount of frequency difference between the DFB-LD wavelength without modulation and the peak of the filter pass-band. Since each of spectral components corresponding to the " 0 "-level and the " 1 "-level is selectively filtered out by Gaussian-shaped filter pass-band of the AWG [22], the ER changes whenever passing through an AWG filter.

We evaluated the upstream power penalty against the laser-to-filter detuned frequency for several values of the RSOA injection power and the incident ER. Both the DFB-LD and the RSOA were directly modulated with $1.25-\mathrm{Gb} / \mathrm{s}$ NRZ data. The DFB-LD wavelength was finely detuned by controlling its temperature, while the center frequencies of the wavelength filters were 


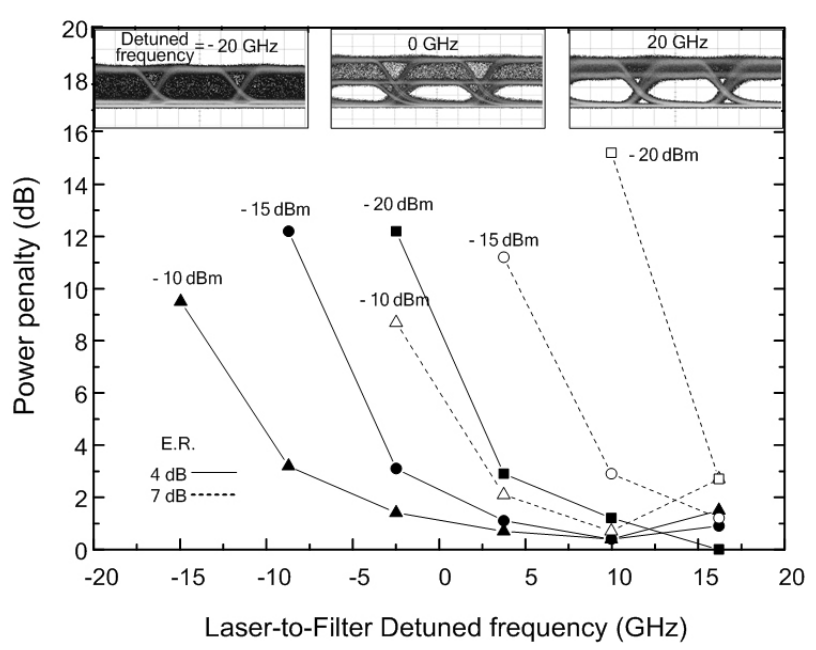

FIG. 12. The measured values of upstream power penalty against the detuned frequency with respect to the injection power levels. The insets are optical eyes for three detuned frequencies of $-20,0$, and $20 \mathrm{GHz}$ with the downstream ER of $7 \mathrm{~dB}$ and the injected power of -10 dBm (Ref. [21]).

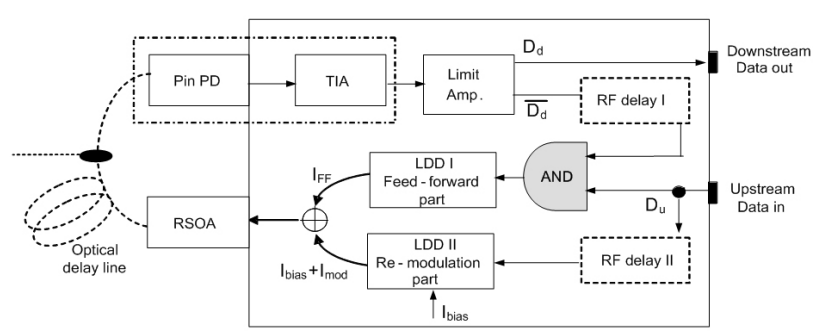

FIG. 13. Block diagram of the proposed optical transceiver with a feed-forward current injection (FFCI) method (Ref. [24]).
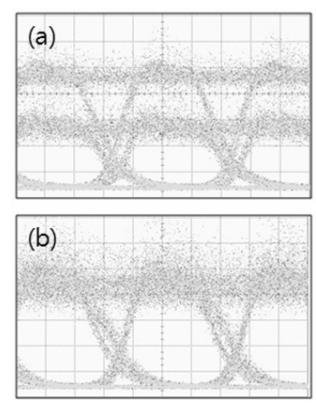

FIG. 14. Improvement of upstream transmission by employing the feed-forward current-injection method. Upstream eye patterns (a)without FFCI and (b)with FFCI, and (c) BER plot with FFCI.

precisely fixed at $193.20 \mathrm{THz}$. The reference BER data for the estimation of the power penalty was also measured when the injection light into the RSOA was continuous wave. The measured values of the upstream power penalty are plotted in Fig. 12 with the upstream optical eyes being inserted in the inset for three different detuned frequencies. The upstream penalty could be maintained below $2 \mathrm{~dB}$ by properly choosing the detuned frequency even with the downstream ER of $7 \mathrm{~dB}$ at the injection power of $-20 \mathrm{dBm}$.

These experimental results clearly indicate that the upstream quality can be improved by intentionally detuning the wavelength of a DFB-LD from AWG passband center wavelength. Using this effect, it is possible to increase the downstream ER in this type of RM scheme while keeping both the downstream and upstream power penalty below an allowable point, for example, $3 \mathrm{~dB}$.

\section{B. Feed-Forward Current Injection}

Another way of improving upstream transmission in the RM schemes is to dynamically vary the RSOA driving current in such a way that the magnitude of the applied modulation current depends on the incident downstream binary signal [23-24]. The corresponding functional block diagram is illustrated in Fig. 13.

The total driving current into an RSOA consists of two parts: (1) amplitude-squeezing part for erasing the incident downstream data and (2) re-modulation part for carrying the upstream data. Here, the driving current for amplitude-squeezing part is fed from the inverted downstream data in the limiting amplifier through the LD driver (LDD)-I. Then, the magnitude of an RSOA gain changes to the opposite direction of the downstream logic level and suppresses the residual downstream signal in the upstream " 1 "-level.

As shown in Fig. 14(a) and (b), the upstream eye pattern with this FFCI technique shows much better eye opening. The corresponding BER plots are shown in Fig. 14(c). Here, the data rate was $1.25 \mathrm{Gbps}$ with a pattern length of $27^{-1}$. The downstream ER was $6 \mathrm{~dB}$ as in other RM schemes. Without counting the polarization-dependence of around $2 \mathrm{~dB}$, the receiver sensitivity with a PIN photo-detector was measured to be around $-27 \mathrm{dBm}$ at the injection power of $-15 \mathrm{dBm}$. When the injection power decreases to $-20 \mathrm{dBm}$, the sensitivity degrades only by $1 \mathrm{~dB}$. The polarization dependence comes from the intrinsic RSOA polarization-dependent property, not from the FFCI technique.

\section{REFLECTION SENSITIVITY IN THE RE-MODULATION SCHEMES}

The optical link of the WDM-PON with the remodulation scheme uses exactly the same wavelength for bidirectional transmission over single optical fiber. It provides distinctive, practical advantages such that multiple-stage remote nodes can be easily implemented using simple combination of add/drop filters, and that there is no requirement for the cyclic wavelength response in the WDM MUX filters. However, the link is 


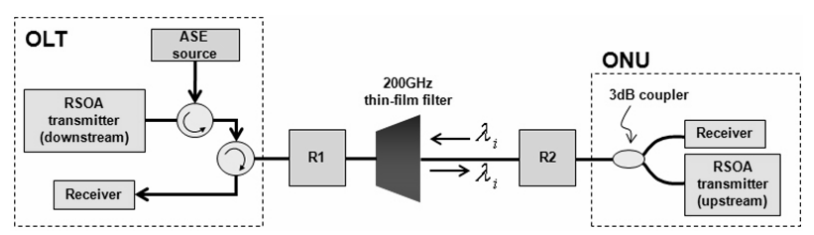

(a)

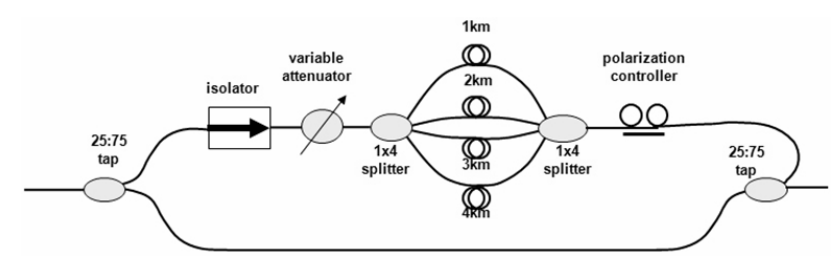

(b)

FIG. 15. (a) Link test setup for emulating reflection effect and (b) the detailed configuration of the reflector $\mathrm{R} 1$ and R2 [Ref. 25]

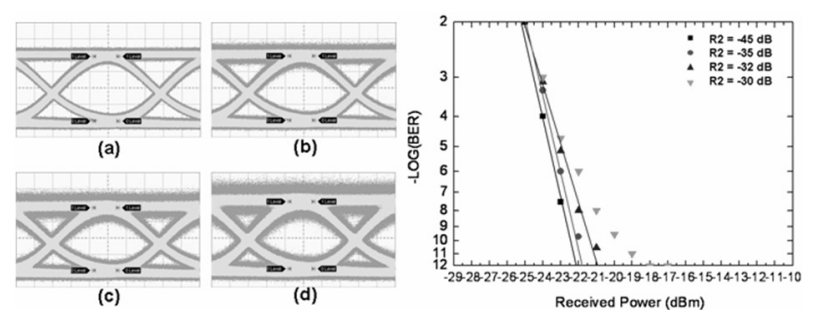

FIG. 16. Optical eye diagrams with various R2 reflections and the corresponding BER plots: (a) $\mathrm{R} 2=-45$ $\mathrm{dB}$, (b) $\mathrm{R} 2=-35 \mathrm{~dB}$, (c) $\mathrm{R} 2=-32 \mathrm{~dB}$, (d) $\mathrm{R} 2=-30 \mathrm{~dB}$. A DFB-LD is used for the seed light.
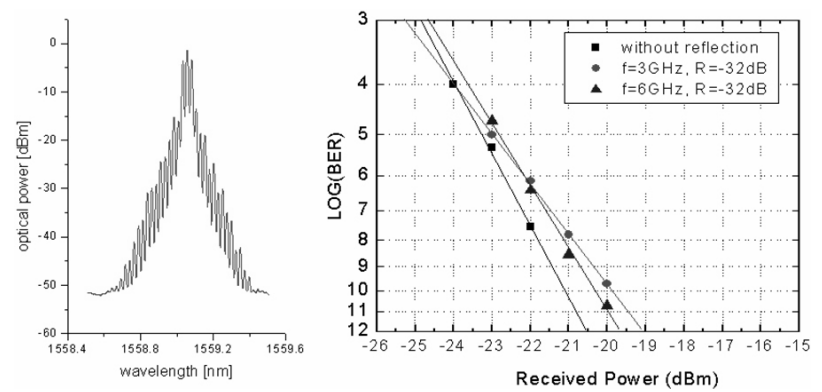

FIG. 17. Spectral broadening of the phase-modulated DFB-LD light and its BER data at $-32 \mathrm{~dB}$ reflection.

vulnerable to parasitic reflections caused either by bad fiber connections or by Rayleigh reflection in the optical fiber. This reflected signal induces beat noise against the intended signal at the receiver [25].

Figure 15(a) shows the test setup for characterizing the effect of reflection on transmission quality. What is different from the previous link structure shown in Fig. 4 is that an RSOA is employed as a colorless transmitter at both sides of OLT and ONU. For downstream transmission, single seed light is placed at the OLT for feeding a continuous WDM light to an OLT RSOA.
Using this downstream signal as a seed light to an ONU RSOA, the upstream signal is generated by the aforementioned re-modulation techniques. Figure 15(b) shows the setup for emulating reflection on the link. The magnitude of link reflection is able to be controlled with the variable attenuator, whereas polarization states of the reflected light being controlled with the polarization controller [25]. In the figure, $\mathrm{R} 1$ is the reflection of the downstream optical signal toward the OLT side, and $\mathrm{R} 2$ is the reflection of the upstream signal toward the ONU side.

In the following two subsections, we investigate qualitatively the reflection sensitivity for two different types of seed light; coherent light from DFB-LD array and spectrum-sliced ASE light (incoherent light). The reason for choosing these light sources is that the reflectioninduced beat noise is dominated by the spectral BW of the seed light [26].

\section{A. DFB-LD Seed Light}

Figure 16 shows the measured optical eyes and the BER plots by increasing R2 from $-45 \mathrm{~dB}$ up to $-30 \mathrm{~dB}$. It can be clearly seen that reflection noise is added to both "1"-level and "0"-level, resulting in a considerable reduction of eye opening. The ER of downstream was $6 \mathrm{~dB}$, and the receiver has sensitivity of $-22 \mathrm{dBm}$ in the absence of reflection. It tends to show an error flow even at $-32 \mathrm{~dB}$ reflection.

Considering the Rayleigh reflection of $-32 \mathrm{~dB}$ in the practical link of $20 \mathrm{~km}$ reach, we need to improve the reflection sensitivity to such a level of allowing error-free transmission at $-32 \mathrm{~dB}$ reflection. One straightforward method is spectral broadening by phase-modulation of the narrow linewidth DFB-LD light. As shown in Fig. 17 , the phase-modulated output spectrum shows a $3-\mathrm{dB}$ BW of around $0.1 \mathrm{~nm}$. The corresponding BER data shows power penalty of $1 \mathrm{~dB}$ without any sign of error floor at $-32 \mathrm{~dB}$ reflection.

\section{B. Spectrum-Sliced ASE Seed Light}

The spectrum-sliced ASE light has much broader linewidth than that of DFB-LD, e.g., $0.8 \mathrm{~nm}$ for a $200 \mathrm{GHz}$ spacing WDM MUX used in the experiment. Considering the receiver $3-\mathrm{dB} \mathrm{BW}$ of around $1 \mathrm{GHz}$ (0.008 $\mathrm{nm}$ in wavelength), the amount of the beat noise which enters the receiver BW is roughly estimated to be 100 times smaller than the total amount of the beat noise. As expected, the improved reflection immunity can be seen from Fig. 18, where there are no noticeable changes in the optical eye diagrams for various reflections. Consequently, the BER plots shows a power penalty of less than $0.5 \mathrm{~dB}$ even at $-30 \mathrm{~dB}$ reflection. 

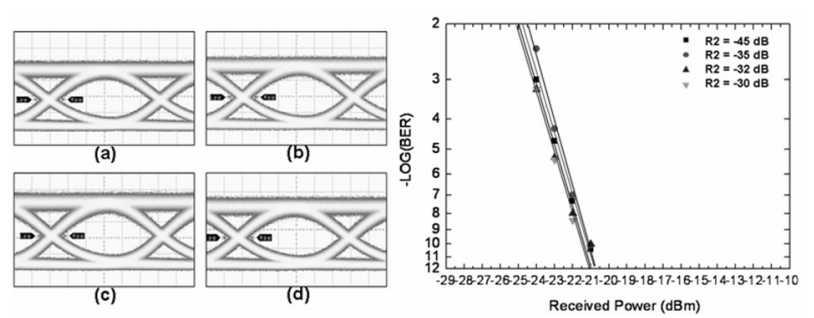

FIG. 18. Optical eye diagrams with various R2 reflections and the corresponding BER plots: (a) R2=-45 dB, (b) $\mathrm{R} 2=-35 \mathrm{~dB}$, (c) R2=-32 dB, (d) R2=-30 dB. Spectrumsliced ASE source with a $3-\mathrm{dB}$ bandwidth of $0.8 \mathrm{~nm}$ was used for the seed light source.

\section{CONCLUSION}

The re-modulation schemes with an RSOA as a colorless transmitter were reviewed in terms of technical achievements for a simplest bi-directional gigabit WDMPON structure. In these schemes, it is essential to efficiently erase the downstream signal residing inside the upstream "1"-level so that the re-modulated upstream signal can be transmitted to the opposite OLT receiver with a least power penalty. To this end, firstly we reviewed the gain-saturation characteristics and its related properties such as ER suppression, noise reduction at low frequency region, and nonlinear spectral distortion. And then, we explained our novel optical/electrical compensation techniques: frequency-detuning and feed-forward current injection.

The frequency-detuning method utilizes the adiabaticchirped output spectrum of a directly-modulated DFBLD in reducing the residual ER by selectively filtering its "0"- or "1"-levels through Gaussian-shaped filter. The FFCI method is to dynamically vary the RSOA driving current in such a way that the magnitude of an RSOA gain changes to the opposite direction of the downstream binary logic level for suppressing the residual downstream signal. These two methods, properly combined with an RSOA gain-saturation property, could be used in enhancing the link loss budget. For example, the downstream ER can be increased up to $6.5 \mathrm{~dB}$ at the injection power of $-17 \mathrm{dBm}$ to a remote RSOA, achieving an error-free bidirectional gigabit transmission over $20 \mathrm{~km}$ fiber.

Finally, we analyzed reflection sensitivity of the remodulated links for using two different types of seed lights: coherent light from DFB-LD array and spectrumsliced ASE light. The experiment showed that a considerable improvement in transmission quality in the presence of $-30 \mathrm{~dB}$ reflection can be achieved by using spectrum-sliced ASE source as the seed light source for the RSOA.

\section{REFERENCES}

[1] B. W. Kim, H. H. Lee, and J. W. Yang, "Access network technologies for broadband subscriber services in Korea: President and future," IEICE Trans. Comm., vol. E86-B, no. 8, pp. 2273-2286, 2003.

[2] S.-J. Park, C.-H. Lee, K.-T. Jeong, H.-J. Park, J.-G. Ahn, and K.-H. Song, "Fiber-to-the-home services based on wavelength-division-multiplexing passive optical network," IEEE J. Lightwave. Tech., vol. 22, no. 11, pp. 2582-2590, 2004.

[3] K. H. Han, E. S. Son, K. W. Lim, H. Y. Choi, S. P. Jung, and Y. C. Chung, "Bi-directional WDM Passive Optical Network using Spectrum-Sliced Light-Emitting Diodes," in Proc. of OFC'2005, Los Angeles, CA, Paper MF98.

[4] H. D. Kim, S. G. Kang, and C.-H. Lee, “A Low-cost WDM source with an ASE injected Fabry-Perot semiconductor laser," IEEE Photon. Technol. Lett. vol. 12, no. 8, pp. 1067-069, 2000.

[5] S.-G. Mun, M.-H. Kim, S.-M. Lee, and C.-H. Lee, “A $240 \mathrm{~km}$ reach DWDM-PON of 8-Gb/s capacity using an optical amplifier," J. Opt. Soc. Korea, vol. 11, no. 3, pp. 93-96, 2007.

[6] G.-Y. Kim and J.-H. Kim, "Wavelength Division Multiplexing- Passive Optical Network Based FTTH Field Trial Test," J. Opt. Soc. Korea, vol. 11, no. 3, pp. 101107, 2007.

[7] S. B. Park, D. K. Jung, D. J. Shin, et al, "Demonstration of WDM-PON with $50 \mathrm{GHz}$ channel spacing employing spectrum-sliced reflective semiconductor optical amplifiers," IEE Electron. Lett., vol. 42, no. 20, pp. 1172-1173, 2006.

[8] S. J. Park, G. Y. Kim, and T. S. Park, "WDM-PON system based on the laser light injected reflective semiconductor optical amplifier," Optical Fiber Technology, vol. 12, no. 2, pp. 162-169, 2006.

[9] M. D. Feuer, J. M. Wiesenfeld, J. S. Perino, C. A. Burrus, G. Raybon, S. C. Shunk, and N. K. Dutta, "Single-port laser-amplifier modulators for local access," IEEE Photon. Technol. Lett., vol. 8, no. 9, pp. 1175-1177, 1996.

[10] W. Hung, C. Chan, L. Chen, and F. Tong, "An optical network unit for WDM access networks with downstream DPSK and upstream remodulated OOK data using injection-locked FP laser," IEEE Photon. Technol. Lett., vol. 15, no. 10, pp. 1476-1478, 2003.

[11] H. Takesue, and T. Sugie, "Wavelength channel data rewrite using saturated SOA modulator for WDM networks with centralized light sources," IEEE J. Lightwave. Tech., vol. 21, no. 11, pp. 2546-2556, 2003.

[12] J. J. Koponen and M. J. Soderlund, "A duplex WDM passive optical network with 1:16 power split using reflective SOA remodulator at ONU," in Proc. of OFC'2004, Los Angeles, CA, Paper MF99, 2004.

[13] N. Buldawoo, S. Mottet, H. Dupont, D. Sigogne, and D. Meichenin, "Transmission experiment using a laser amplifier-reflector for DWDM access network, ” in Proc. of ECOC'98, Madrid, Spain, pp. 196-199, 1998.

[14] P. Healey, P. Townsend, C. Ford, L. Johnston. P. Townley, I. Lealman, L. Rivers, S. Perrin, and R. Moore, "Spectral slicing WDM-PON using wavelength-seeded 
reflective SOAs," IEE Electron. Lett., vol. 37, no. 19, pp. 1181-1182, 2001.

[15] W. R. Lee, M. Y. Park, S. H. Cho, J. Lee, C. Kim, G. Jeong, and B. W. Kim, "Bidirectional WDM-PON based on gain-saturated reflective semiconductor optical amplifiers," IEEE Photon. Technol. Lett., vol. 17, no. 11, pp. 2460-2462, 2005.

[16] K. Sato and H. Toba, "Reduction of mode partition noise by using semiconductor optical amplifiers," IEEE J. Quantum Elec., vol. 7, no. 2, pp. 328-333, 2001.

[17] S. G. Mun, J. H. Moon, H. K. Lee, J. Y. Kim, and C.-H. Lee, "A WDM-PON with a $40 \mathrm{~Gb} / \mathrm{s}(32 \times 1.25$ $\mathrm{Gb} / \mathrm{s})$ capacity based on wavelength-locked Fabry-Perot laser diodes," Opt. Exp., vol. 16, no. 15, pp. 1136111368, 2008.

[18] A. D. McCoy, P. Horak, B. C. Thomsen, M. Ibsen and D. J. Richardson, "Noise suppression of incoherent light using a gain-saturated SOA: Implications for spectrumsliced WDM systems," IEEE J. Lightwave. Tech., vol. 23, no. 8, pp. 2399-2409, 2005.

[19] M. Munroe, J. Cooper, and M. G. Raymer, "Spectral broadening of stochastic light intensity-smoothed by a saturated semiconductor optical amplifier," IEEE J. Quantum Elec., vol. 34, no. 3, pp. 548-551, 1998.

[20] G. V. Agrawal and N. A. Olsson, "Self-phase modulation and spectral broadening of optical pulses in semiconductor laser amplifiers," IEEE J. Quantum Elec., vol. 25, no.
11, pp. 2297-2306, 1989.

[21] W. R. Lee, S. H. Cho, M. Y. Park, J. Lee, C. Kim, G. Jeong, and B. W. Kim, "Frequency detuning effects in the loop-back WDM-PON employing gain-saturated RSOAs," IEEE Photon. Technol. Lett., vol. 18, no. 13, pp. 1436-1438, 2006.

[22] J. Downie, I. Tomkos, N. Antoniades, and A. Boskovic, "Effects of filter concatenation for directly modulated transmission lasers at 2.5 and $10 \mathrm{~Gb} / \mathrm{s}$, IEEE J. Lightwave. Tech., vol. 20, no. 2, pp. 218-228, 2002.

[23] E. Conforti, C. M. Gallep, S. H. Ho, A. C. Bordonalli and S. Kang, "Carrier reuse with gain compression and feed-forward semiconductor optical amplifier ," IEEE Trans. Microwave Theory and Techniques, vol. 50, no. 1, pp. 77-81, 2002.

[24] W. R. Lee, S. H. Cho, M. Y. Park, J. Lee, C. Kim, G. Jeong, and B. W. Kim, "Optical transceiver employing an RSOA with feed-forward current injection,” in Proc. of OFC2007, Anaheim, California, USA.

[25] M. Fujiwara, J.-I. Kani, H. Suzuki, and K. Iwatsuki, "Impact of backreflection on upstream transmission in WDM single-fiber loopback access networks," IEEE J. Lightwave. Tech., vol. 24, no. 2, pp. 740-746, 2006.

[26] H. W. Hu and H. Anis, "Degradation of bi-directional single fiber transmission in WDM-PON due to beat noise," IEEE J. Lightwave. Tech., vol. 26, no. 8, pp. 870-881, 2008. 